\title{
El proceso de la reconstrucción de la red personal de los inmigrantes: una descripción longitudinal
}

\author{
The reconstruction of personal networks among immigrants: \\ A longitudinal description
}

\author{
Miranda J. Lubbers \\ Universidad Autónoma de Barcelona \\ mirandajessica.lubbers@uab.cat (ESPAÑA) \\ José Luis Molina \\ Universidad Autónoma de Barcelona \\ joseluis.molina@uab.es (ESPAÑA)
}

Recibido: 12.012011

Aceptado: 06.05.2013

\section{RESUMEN}

Estudios anteriores han mostrado que la red personal puede ser una fuente de apoyo social para inmigrantes, pero no es una entidad estática. En este artículo describimos un estudio longitudinal sobre el cambio en las redes personales de inmigrantes, realizado entre 2004-2008 en cuatro colectivos de inmigrantes relativamente recientes en Cataluña (en total $\mathrm{N}=70$ ). Las redes se han medido en dos oleadas, ambas post-migratorias, con un intervalo de tiempo de 1-3,3 años. Comparando las redes en los dos momentos, analizamos el grado de renovación en las redes, las tendencias generales del cambio en términos de su composición e investigamos las diferencias en estas tendencias en base de género, grupo étnico y años de residencia. Los resultados indican que las redes son poco estables en general, pero que a pesar de ello, la composición media de las redes no cambia apenas, salvo por lo que se refiere a un ligero aumento del número de compatriotas residentes en España y un descenso del número de personas residiendo en el país de origen. En general, no observamos un aumento significante del número de españoles en las redes personales de los inmigrantes.

EMPIRIA. Revista de Metodología de Ciencias Sociales. N. ${ }^{\circ}$ 26, julio-diciembre, 2013, pp. 63-88. ISSN: 1139-5737, DOI: 10.5944/empiria.26.7153 


\title{
PALABRAS CLAVES
}

Redes personales, inmigrantes, adaptación, análisis longitudinal.

\begin{abstract}
Past studies indicated that the personal networks can be a source of social support for immigrants, but it is not a static entity. In this article, we describe a longitudinal study into the change in personal networks of immigrants, performed between 2004-2008 in four groups of immigrants with relatively recent residence in Catalonia, Spain (in total $\mathrm{N}=70$ ). The networks were measured twice, both waves post-migratory, with a period of 1,0-3,3 years in between. Comparing the networks of the two waves, we analyzed the turnover in networks, the general tendencies with respect to the changes in composition of the networks, and we investigated differences in these tendencies based on gender, ethnic group and years of residence. The results indicated that networks are relatively unstable, but that nevertheless, the average composition of the networks hardly changes, apart from a small increase in the number of co-ethnics residing in the host society, and a decrease in the number of persons residing in the country of origin. In general, we did not observe a significant tendency toward the incorporation of Spaniards in the networks.
\end{abstract}

\section{KEYWORDS}

Personal networks, immigrants, adaptation to host society, longitudinal analysis

\section{INTRODUCCIÓN}

Diferentes estudios en el ámbito de la migración internacional han documentado ampliamente el hecho que las redes sociales influyen en la decisión de emigrar, que proporcionan dinero e información para facilitar el traslado y que proveen a continuación alojamiento, empleo, información y apoyo emocional, entre otros recursos (e.g. Boyd 1989; Carnet, 2011; Palloni et al. 2001; Poros, 2011). En este sentido, la red social puede llegar a ser una fuente de recursos importante para los inmigrantes. Mientras que estudios anteriores trataban la red social implícitamente como una entidad estática, aportaciones recientes resaltan que tanto las redes de los inmigrantes como los recursos que fluyen por ellas cambian durante el proceso de la migración y la instalación (e.g. Gill \& Bialski, 2011; Ryan, 2011; Carnet, 2011; Wierzbicki, 2004). Esta dinámica implica que los recursos sociales no siempre están disponibles, como se sugería inicialmente.

EMPIRIA. Revista de Metodología de Ciencias Sociales. N. 26, julio-diciembre, 2013, pp. 63-88. ISSN: 1139-5737, DOI: 10.5944/empiria.26.7153 
Por el contrario, mientras la necesidad de apoyo social suele ser más elevada durante el proyecto migratorio, la red personal suele sufrir simultáneamente un serio proceso de desorganización, con todas las implicaciones que ello conlleva. Así, una parte de las relaciones personales ubicadas en el país de origen suele desaparecer a corto plazo, particularmente los lazos más débiles mientras que las relaciones que se mantienen en origen tienen que reinventarse en forma y contenido desde un nuevo escenario transnacional (Ávila Molero, 2008). En general, son los lazos con familiares localizados en origen los que mejor sobreviven a la separación, a pesar de que la frecuencia de contacto suele reducirse (e.g., Lubbers et al., 2010; Vacca, 2013). Aunque las relaciones que los inmigrantes mantienen en origen pueden continuar dando apoyo al inmigrante (y vice versa), a causa de la distancia y el desconocimiento de la sociedad de acogida, estas relaciones ya no suelen ser capaces de proporcionar todos los tipos de apoyo que el inmigrante necesita, particularmente los tipos de apoyo que requieren presencia. En este sentido se ha constatado que el apoyo social localmente disponible es crucial para facilitar la instalación en el país de recepción. En el momento de la llegada, los inmigrantes solamente conocen a las personas que les han acompañado en el trayecto migratorio y las personas en destino con las que ya tenían un cierto contacto (directo o indirecto) antes de trasladarse. Son estas personas las que les puedan brindar apoyo, pero incluso estas relaciones suelen cambiar en sus contenidos a causa del proyecto migratorio y las condiciones específicas del contexto de instalación (Wierzbicki, 2004; Menjívar, 2000).

Dada la importancia fundamental del apoyo social localmente disponible, es relevante entender cómo los inmigrantes reconstruyen a sus redes una vez instalados en destino. En un estudio basado en grupos focales con inmigrantes peruanas residentes de Sevilla, Maya Jariego et al. (1999) observaron cuatro estrategias para la reconstrucción reticular en la sociedad española: (1) la concentración de funciones en unos pocos vínculos con compatriotas, (2) la utilización de grupos de compatriotas - en lugar de individuos - como unidades de apoyo, (3) el reagrupamiento familiar y (4) la incorporación de españoles. Comparando estas cuatro estrategias, Maya Jariego y sus compañeros mencionan que «si las dos primeras son estrategias de compensación y aprovechamiento del reducido tamaño del entorno personal, las dos últimas posibilitan el crecimiento de la red de apoyo, así como la conformación de una estructura más compleja, con una composición más equilibrada y con proveedores menos cargados de funciones». Igualmente, las diferentes estrategias suelen ser utilizadas en diferentes fases de la trayectoria migratoria. En una primera fase tras el traslado, los inmigrantes suelen tener pocas relaciones locales, sobre todo con compatriotas. En muchos casos estos compatriotas proporcionan un apoyo polivalente en los primeros años, aunque se ha documentado que el apoyo de los compatriotas es contingente con las condiciones materiales y físicas de las relaciones sociales y muchas veces falla o provoca conflictos (Mahler, 1995; Menjívar, 1997). En general, se asume que la red se reconstruye poco a poco incluyendo en primer lugar nuevas relaciones con compatriotas residiendo en destino, con los que los inmigrantes se comunican con más facilidad y comparten más valores y experiencias. En 
segundo lugar, se pueden incorporar ciudadanos nativos de la sociedad de residencia (Lynam, 1985) e inmigrantes procedentes de otros países. A pesar de que las teorías de asimilación han sido muy debatidas en las últimas décadas, la existencia de vínculos con españoles en las redes personales de los inmigrantes aún suele considerarse como un indicador del grado de incorporación social en el nuevo entorno, asociado con otros resultados positivos de integración (e.g., Martínez García et al., 2002; Martinovic, 2013; Martinovic, Van Tubergen, \& Maas, 2009; Valenta, 2009; Vervoort, 2012; Wierzbicki, 2004).

Desde luego, hay mucha variación entre inmigrantes tanto en el tipo de estrategia (o proceso) como en el ritmo con el que se llevan a cabo estos procesos. Esta variación se puede explicar en parte en base de características individuales o colectivas. En este sentido, la medida en que un inmigrante logra incorporar a nuevas personas en su red depende de características individuales como por ejemplo sus habilidades sociales y lingüísticas o el tipo de migración, así como de las características del contexto ecológico de origen, instalación y la intersección entre ambos. Así mismo, también es fundamental la estructura y la composición social de los diferentes entornos sociales en los que el individuo participa (e.g., el mercado laboral, el barrio, las asociaciones; $C f$. Feld, 2004). En este sentido, los procesos de reconstrucción reticular están muy encuadrados en las características de los niveles micro, meso y macro. Estudios cualitativos ya han proporcionado informaciones más holísticas sobre la significación de relaciones sociales para determinados grupos de inmigrantes en determinados lugares tomando en cuenta estos contextos, sin trazar explícitamente al conjunto de las relaciones, es decir, la red (e.g., Eve, 2010; Gill \& Bialski, 2011; Poros, 2011; Ryan, 2011). No obstante, para avanzar nuestro conocimiento sobre la reconstrucción reticular entre inmigrantes, también es importante averiguar las tendencias medias en los cambios de las redes entre migrantes procedentes de diferentes países y con diferentes características. Varios estudios ya han avanzado este conocimiento significativamente (e.g., Martinovic et al., 2009; De Miguel Luken \& Tranmer, 2010; Liu, 2011). En el presente artículo, proponemos una metodología que pueda complementar los estudios anteriores de dos formas. En primer lugar, proponemos un diseño longitudinal para poder comparar las redes existentes en dos momentos diferentes. Esta metodología es esencial para entender el proceso de reconstrucción. Así por ejemplo, la correlación entre el tiempo de residencia y la composición de la red observada en estudios transversales suelen interpretarse con frecuencia como un indicador de tendencia temporal, pero éste no es necesariamente un argumento válido. Una explicación alternativa sería por ejemplo que la cohorte migratoria está relacionada con diferencias en las condiciones iniciales del contexto local (e.g., el número de compatriotas en destino), lo cual puede explicar la composición de la red (e.g., el porcentaje de compatriotas en destino en la red) aún si las redes no cambian en el tiempo.

En segundo lugar, proponemos una operacionalización más amplia del concepto «red personal». En general, la literatura que estudia las redes personales 
suele limitarse al estudio de los lazos más fuertes. Hay varias definiciones ${ }^{1}$ e indicadores de la fuerza de las relaciones, pero quizás el mejor indicador (Marsden \& Campbell, 1984) es el de la percepción de que la relación es íntima y especial. El énfasis en los lazos fuertes en el estudio de las redes de inmigrantes se justifica porque las personas conectadas por lazos fuertes están más motivadas y más disponibles para proveer el apoyo material y emocional (Granovetter, 1973). En este sentido, la literatura inicial sobre redes y emigración asume que las relaciones fuertes con compatriotas forman conglomerados densos y cerrados y son las que proporcionan más apoyo social (e.g., Portes, 1995; Portes \& Sensenbrenner, 1997; Sanders, Nee \& Sternau, 2002). No obstante, los lazos «débiles» también aportan recursos importantes, como ha señalado Granovetter en su estudio pionero «The strength of weak ties» (1973). Los lazos débiles proporcionan acceso a una mayor variedad de información por ser mucho más numerosos, más heterogéneos y menos conectados con los demás lazos del individuo y por lo tanto pueden ser un recurso para fines instrumentales como la búsqueda de vivienda o empleo. En términos de la reinstalación de inmigrante, el acceso a los lazos débiles (particularmente con nativos) se ha descrito como un elemento clave para diversificar la red, para acceder a nuevas informaciones (e.g., Stanton-Salazar et al., 1995), para aprender nuevos roles (Aroian, 1992), sentirse a gusto en la comunidad (Rose, Carrasco \& Charboneau, 1998) y como símbolo de aceptación que ayuda a construir identidades más inclusivas (Valenta, 2009). Varios autores (e.g., Valenta, 2009) han sugerido que es la mezcla de relaciones fuertes y relaciones débiles que hacen de «puente» la más adecuada para la adaptación en la sociedad de acogida. Resumiendo: varios estudios han resaltado la importancia que tiene medir redes más amplias para poder estudiar el arraigo relacional de los inmigrantes (véase también Liu, 2011; Pfeffer \& Parra, 2009; Ryan, 2011; Wilson, 1998).

En el presente artículo presentamos los resultados de un estudio longitudinal de redes personales realizado en cuatro colectivos de inmigrantes (argentinos, dominicanos, marroquíes y senegambianos) instalados relativamente recientemente en la provincia de Barcelona. Para cada persona, se ha recogido la red personal activa de 45 miembros en dos momentos posmigratorios, con un intervalo de tiempo de 2,1 años de media. Comparando la composición de la red en los dos momentos, respondemos a las siguientes preguntas: primero, en promedio y sobre el período mencionado anteriormente ¿en qué medida se renuevan las

${ }^{1}$ Granovetter (1973) definió la fuerza de un lazo intuitivamente como «una (probablemente lineal) combinación del tiempo, la intensidad emocional, intimidad (confianza) y los servicios recíprocos que caracterizan a dicho vínculo» (la traducción es de García Verdasco, http://pendientedemigracion.ucm.es/info/pecar/Articulos/GRANOVETTER2.pdf). Publicaciones posteriores han criticado esta definición y/o los indicadores incluidos (e.g., Marsden y Campbell, 1984). Igualmente, varios indicadores o combinaciones de ellos se han utilizado en estos estudios, como la intensidad emocional, la duración de la relación, la frecuencia de contacto, la reciprocidad y multiplicidad de la relación (e.g., Wellman \& Wortley, 1990) y la estabilidad de la relación (Marsden \& Campbell, 2012). En todo caso Marsden y Campbell (1984) consideran que la intensidad emocional es la variable que refleja mejor el concepto.

EMPIRIA. Revista de Metodología de Ciencias Sociales. N. ${ }^{\circ}$ 26, julio-diciembre, 2013, pp. 63-88. ISSN: 1139-5737, DOI: 10.5944/empiria.26.7153 
redes en el corto plazo? En segundo lugar investigamos si se produce un proceso de arraigo relacional local en las redes. En particular, nos preguntamos ien promedio, cómo cambia la composición de las redes en términos del lugar de origen y de residencia de los miembros de la red en este periodo? ¿Qué ocurre con la fuerza de los lazos? Describimos tanto las tendencias medias como la heterogeneidad en estas tendencias generada por género, país de origen y tiempo de residencia en España. En general, suponemos que el número medio de relaciones locales, el número de españoles en particular y/o la intensidad de los lazos y la frecuencia del contacto con ellos aumentan con el tiempo, mientras que el número medio de lazos en origen y la intensidad emocional y frecuencia de contacto con estos lazos disminuye. En segundo lugar, distinguimos en qué medida las tendencias generales son diferentes para las categorías de las diferentes variables. El tiempo de residencia es una variable esencial pues nos informa sobre la fase en el proyecto migratorio en el que el inmigrante se encuentra. Suponemos que los cambios serán más grandes para los inmigrantes llegados recientemente por el proceso de desorganización reticular inicial. El país de origen incide tanto en las diferencias observadas entre los colectivos (e.g., diferencias culturales, niveles de discriminación experimentada, la presencia de compatriotas en la sociedad catalana), como en las diferencias en las características individuales (e.g., nivel de educación, clase social en origen, tipo de migración, etc.). Estas diferencias afectan profundamente a la posibilidad de formar relaciones de diferentes tipos en España (e.g., Martínez García, García Ramírez \& Maya Jariego, 1999; Miguel Luken \& Tranmer, 2010). Para poder estudiar los procesos de reconstrucción de redes en general, nos hemos centrado en diferentes países de origen, pero también describimos las tendencias generales. Suponemos por ejemplo que es más fácil para los argentinos aumentar el número de españoles en su red a lo largo del tiempo que para otros colectivos, dado su nivel de aceptación por parte de la sociedad catalana y por sus niveles de educación y estatus ocupacionales más altos en general. Por último, varios investigadores también han insistido en tener el género en cuenta a la hora de estudiar los procesos migratorios (e.g., Hondagneu-Sotelo \& Cranford, 1999; Hagan, 1998). Estudios previos han mostrado que los hombres y las mujeres forman y organizan sus relaciones sociales de formas diferentes (e.g., Szell \& Turner, 2013), produciendo por ejemplo redes más homogéneas entre mujeres y redes más diversificadas entre hombres (e.g., Marsden, 1987; Kürtosi, 2008). Suponemos por lo tanto que los hombres muestran cambios más significativos en sus redes que las mujeres. Reconocemos que hay muchas otras variables afectando potencialmente a los procesos de reconstrucción de la red, como la inserción laboral y el estatus socio-económico, el nivel educativo, la edad, la religión, el estatus marital, el tipo de migración, el tener hijos o no, las cargas familiares, trayectorias personales, etcétera. No obstante, dado que nuestro objetivo consiste en esbozar las líneas generales de los procesos de la reconstrucción de la red personal, nos hemos centrado en las variables que hemos creído más importantes para explicar este proceso.

EMPIRIA. Revista de Metodología de Ciencias Sociales. N. ${ }^{\circ}$ 26, julio-diciembre, 2013, pp. 63-88. ISSN: 1139-5737, DOI: 10.5944/empiria.26.7153 


\section{DEFINICIÓN DE RED PERSONAL}

Definimos la red personal como el conjunto de relaciones sociales activas de un individuo focal, que provienen de los diversos contextos en que el individuo participa o ha participado (por ejemplo, la familia, el barrio, la empresa, una asociación,...). Con el término «relación activa» nos referimos a la definición de Dunbar (p.ej., Dunbar \& Spoor, 1995; Zhou, Sornette, Hill \& Dunbar, 2005), es decir, a las relaciones con personas que el individuo focal considera relaciones personales, para las que se esfuerza en mantener el contacto y con los que ha tenido contacto por lo menos alguna vez en los últimos dos años. Esta red, según Dunbar, tiene un tamaño medio de unas 45-50 personas ${ }^{2}$, pero este tamaño puede variar entre individuos.

Figura 1. Los niveles jerárquicos de proximidad emocional en la red personal y el número estimado de personas en cada nivel, según Dunbar (Dunbar \& Spoor, 1995; Zhou, Sornette, Hill, \& Dunbar, 2005)

go

grupo de apoyo \pm 5

grupo de simpatía \pm 15

red activa / cercana \pm 50

red personal \pm 150

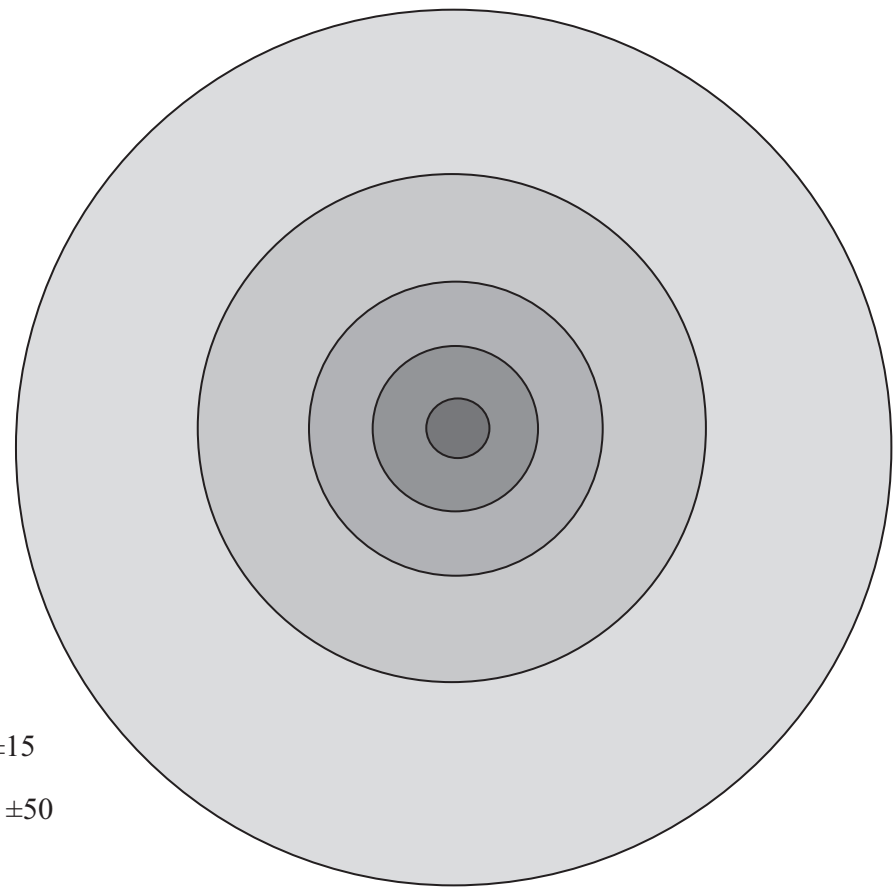

Estudios anteriores solían capturar una parte mucho más pequeña de la red personal, a menudo por razones logísticas, midiendo solamente las 5-10 relaciones sociales más íntimas, las que se conocen en la literatura como el grupo de

${ }^{2}$ En la literatura no existe un acuerdo sobre el tamaño preciso de la red personal, y varios investigadores han llegado a números muy discrepantes.

EMPIRIA. Revista de Metodología de Ciencias Sociales. N. ${ }^{\circ}$ 26, julio-diciembre, 2013, pp. 63-88. ISSN: 1139-5737, DOI: 10.5944/empiria.26.7153 
apoyo. En nuestra opinión, para la medición de la reconstrucción de la red de los inmigrantes, es esencial incluir no solamente los lazos más fuertes e íntimos, sino también a los lazos que son significativos para el individuo focal pero más débiles, siendo éstos como ya hemos mencionado más numerosos y heterogéneos que los lazos más íntimos (véase la Figura 1 para los niveles jerárquicos asumidos en la red activa). Es razonable suponer que las relaciones que los inmigrantes adquieren con españoles no son inmediatamente las más íntimas; no obstante, con el tiempo se pueden intensificar. Además, si los encuestados se ven forzados a nombrar por ejemplo 5 nombres, es muy probable que se ignore una parte de sus lazos fuertes. Para capturar una parte más grande de la red personal, varios investigadores han utilizado generadores de nombres más elaborados y recientemente este método se ha aplicado en varios estudios de inmigrantes en España (p.ej., Domínguez \& Maya Jariego, 2008; Lubbers, Molina, \& McCarty, 2007; Lubbers et al., 2010; Molina, Bolíbar \& Cruz, 2011; Molina, Lerner \& Gómez Mestres, 2008). Dicho esto, nuestro estudio es - por lo que sabemos- el único estudio cuantitativo sobre las redes personales amplias de inmigrantes con un diseño longitudinal orientado a capturar el proceso de la reconstrucción de la red.

\section{EL CONTEXTO DE INMIGRACIÓN EN LA PROVINCIA DE BARCELONA ${ }^{3}$}

El estudio se ha centrado en la provincia de Barcelona en el periodo 2004-2006. En este periodo, el porcentaje de extranjeros instalados en la provincia de Barcelona extranjero pasó del 9,2\% (2004) al 12,2\% (2006) siendo actualmente del 14,3\% (2012; datos de IDESCAT en base del padrón). Dichos porcentajes son ligeramente inferiores a los porcentajes para la población total de Cataluña. Los cinco grupos incluidos en nuestra muestra son marroquíes (el 22,3\% y el $20,6 \%$ de la población extranjera en Barcelona en el 2004 y en el 2006 respectivamente), argentinos ( $4,7 \%$ y $4,2 \%)$, senegaleses $(1,1 \%$ y $1,4 \%)$, gambianos $(1,7 \%$ y $1,6 \%)$ y dominicanos $(0,03 \%$ y $0,02 \%)$. El colectivo marroquí es el más numeroso y antiguo de los que están ubicados en Barcelona. En el 2004, el 64\% de los marroquíes en la provincia de Barcelona eran hombres. La migración de los Senegaleses y Gambianos empezó en los años setenta y es mayoritariamente masculina (en Barcelona el $81 \%$ de los senegaleses y el $74 \%$ de los gambianos eran hombres en el año 2004). En cambio, la emigración de dominicanos es mayoritariamente femenina (en Barcelona el 57\% de las personas dominicanas eran mujeres en el 2004). Los tres grupos suelen insertarse en los segmentos bajos del mercado laboral. Los argentinos llegaron a Catalunya en los años setenta y en oleadas posteriores debido a la crisis económica en Argentina disponiendo de una distribución numérica equilibrada de hombres y mujeres y ocupando empleos mucho más diversos en términos de estatus laboral.

\footnotetext{
3 Todos los datos en esta sección son datos de IDESCAT en base del Padrón del 2004, 2006, y 2012 .
}

EMPIRIA. Revista de Metodología de Ciencias Sociales. N. ${ }^{\circ}$ 26, julio-diciembre, 2013, pp. 63-88. ISSN: 1139-5737, DOI: 10.5944/empiria.26.7153 


\section{DATOS}

\section{Muestra}

La primera oleada de los datos fue recogida en el marco del proyecto financiado por la NSF 4 , «Development of a social network measure of acculturation and its application to immigrant populations in South Florida and Northeastern Spain». Entre el 2004 y el 2006, se entrevistaron a 301 inmigrantes en la provincia de Barcelona pertenecientes a 4 colectivos: argentinos, dominicanos, marroquíes y senegaleses/gambianos. El muestreo se realizó con la técnica de bola de nieve, partiendo de múltiples «semillas» por país de origen. A continuación, en el marco del proyecto ECRP financiado por la ESF y el MEC ${ }^{5}$ «Dynamics of actors and networks across levels: individuals, groups, organizations and social settings», se seleccionaron 77 casos para realizar una segunda entrevista en el 2007-2008. Esta entrevista tuvo lugar por media 2,1 años después de la primera $(D T=0,7)$. En este artículo nos basamos en los 77 encuestados para quienes disponemos de dos medidas de sus redes personales. En total, 25 de ellos son argentinos (32\%), 15 dominicanos (19\%), 21 marroquíes (27\%) y 16 son senegaleses o gambianos (21\%). Las entrevistas con personas procedentes de Argentina se han realizado en Barcelona, Vic y otras localidades cercanas a Vic. Las entrevistas con personas de la República Dominicana, de Senegal y Gambia (Diola, Mandinga, Wolof y Bambara) y de Marruecos (Amazigh) se han realizado sobre todo respetivamente en Hospitalet, en Mataró y en Vic y localidades próximas. La residencia media en España es de 5,4 años en la primera oleada y el porcentaje de mujeres es $43 \%$. Como muestra la Tabla 1, los promedios de estas variables difieren bastante entre los cuatro colectivos, debido en parte a las diferentes características migratorias de cada colectivo y en parte al muestreo.

Tabla 1. Descripción de la muestra

\begin{tabular}{lcccc}
\hline \multicolumn{1}{c}{ Colectivo } & N total & N válido & $\begin{array}{c}\text { Media años de } \\
\text { residencia en Espana } \\
\text { en la primera oleada } \\
\text { (y DT) }\end{array}$ & $\begin{array}{c}\text { Porcentaje } \\
\text { de mujeres }\end{array}$ \\
\hline Argentinos & 25 & 24 & $4,3(3,0)$ & $67 \%$ \\
Dominicanos & 15 & 13 & $5,2(2,9)$ & $31 \%$ \\
Marroquíes & 21 & 18 & $9,6(6,7)$ & $56 \%$
\end{tabular}

${ }^{4}$ National Science Foundation, award n. ${ }^{\circ}$ BCS-0417429, dirigido por Christopher McCarty en los Estados Unidos y José Luis Molina en España.

${ }^{5}$ El European Collaborative Research Project (ECRP) financiado por la European Science Foundation y el Ministerio de Ciencia y Tecnología (SEJ2005-25683-E, SEJ2007-29468-E, 05_ECRP_FP026), dirigido por Tom Snijders; el sub-proyecto español fue dirigido por José Luis Molina.

EMPIRIA. Revista de Metodología de Ciencias Sociales. N. ${ }^{\circ}$ 26, julio-diciembre, 2013, pp. 63-88 ISSN: 1139-5737, DOI: 10.5944/empiria.26.7153 


\begin{tabular}{lcccc}
\hline Colectivo & N total & N válido & $\begin{array}{c}\text { Media años de } \\
\text { residencia en España } \\
\text { en la primera oleada } \\
\text { (y DT) }\end{array}$ & $\begin{array}{c}\text { Porcentaje } \\
\text { de mujeres }\end{array}$ \\
\hline $\begin{array}{l}\text { Senegaleses/ } \\
\text { Gambianos }\end{array}$ & 16 & 15 & $1,8(1,3)$ & $0 \%$ \\
\hline Total & 77 & 70 & $5,4(4,9)$ & $43 \%$ \\
\hline
\end{tabular}

\section{Procedimiento}

Las entrevistas se organizaron en dos partes. La primera parte consistió de una encuesta con 4 módulos, en el siguiente orden: (1) preguntas sobre el encuestado (ego); (2) un generador de nombres para delinear la red personal; (3) preguntas sobre cada uno de los miembros de la red (alteri), para determinar la composición de la red y (4) una pregunta repetida para cada pareja de miembros sobre la existencia o no de un lazo entre ellos con el propósito de determinar la estructura de la red. Los datos de la encuesta se recogieron en entrevistas personales asistidas por ordenador con el software Egonet ${ }^{6}$. Una vez finalizada la parte de encuesta, se visualizó la red delante del encuestado y en base a esta visualización se inició una entrevista semi-estructurada sobre la red personal. Esta parte fue grabada y forma una parte esencial de la interpretación y validación de las redes recogidas. Las sesiones completas duraron de media entre una hora y media y dos horas aproximadamente.

En el primer módulo recogimos datos sobre el encuestado y su proceso migratorio, entre otros los datos que usamos en este artículo sobre su sexo, su país de origen y los años de residencia en España.

\section{La identificación de la red personal}

Para identificar la red personal, se pidió a cada persona entrevistada una lista de 45 contactos activos mediante un generador de nombres abierto:

«Por favor, escriba una lista de 45 personas que Usted conozca por su nombre y viceversa. Puede ser cualquier persona. Intente incluir gente que sea próxima e importante para Usted. También puede incluir personas que pueden no ser tan cercanas pero que acostumbra a ver mucho. Puede ayudarle pensar en diferentes grupos de personas en diferentes lugares. Escriba el nombre y el apellido de forma abreviada para que solamente Usted pueda reconocer a las personas. Es importante que no abrevie demasiado para poder reconocerlas más tarde. Por ejemplo: Mig Cervan por "Miguel de Cervantes"».

\footnotetext{
${ }^{6}$ La última versión de EgoNet está disponible libremente en Sourceforge.
}

http://sourceforge.net/projects/egonet/.

EMPIRIA. Revista de Metodología de Ciencias Sociales. N. ${ }^{\circ}$ 26, julio-diciembre, 2013, pp. 63-88. ISSN: 1139-5737, DOI: 10.5944/empiria.26.7153 
Este generador de nombres tiene la ventaja que permite recoger tanto lazos fuertes como lazos relativamente débiles. La práctica de fijar el número de contactos a 45 no nos permite ver el crecimiento del tamaño de la red con el tiempo, lo que es un inconveniente, pero asegura que las redes sean más comparables entre sí. Estudios anteriores han mostrado que el número de personas nominadas por un encuestado no es una estimación fiable del tamaño de la red, pues depende de la energía del encuestado, el nivel de colaboración, su memoria, tendencias de respuesta y de las habilidades del entrevistador. Además, muchas características de la red (por ejemplo densidad, porcentaje de familiares, fuerza media de los lazos) dependen del tamaño de la red. Si cada entrevistado nombra a un número fijo de personas, las características de las redes son más comparables. Para una justificación de la metodología y una discusión de los sesgos cognitivos ver McCarty (2002), McCarty et al. (2007) y Molina et al. (2007) respectivamente. Es importante subrayar que el generador captura tanto a personas en la sociedad de residencia como a personas en la sociedad de origen.

\section{Medidas de la composición de la red}

Una vez que se ha generado una lista de alteri, se pasa a realizar preguntas sobre cada alter. En la segunda oleada, el entrevistador y el entrevistado comparan conjuntamente esta lista con la lista de miembros de red recogida en la primera oleada y completan la variable «repetido» con «sí» o «no» para cada miembro de red de la segunda oleada. Esta práctica nos permite distinguir entre lazos continuos (miembros que han sido nombrado en ambas oleadas), lazos antiguos (miembros que han sido nombrado en la primera oleada pero no en la segunda) y lazos nuevos (miembros que han surgido por primera vez en la segunda oleada). Así podemos investigar el cambio en la red, no solamente al nivel de la red en su conjunto, sino también al nivel de relaciones diádicas.

En ambas oleadas, recogimos datos sobre el país de origen y el país de residencia. En base de ellos, creamos 4 tipos de miembros de red (véase por ejemplo Molina et al., 2008): (1) españoles —aquellos miembros de red nacidos en España y que residen en España (es decir en Cataluña u en otras partes de España); (2) compatriotas residiendo en destino- miembros cuyo país de residencia es España pero el país de origen es otro, (3) personas residiendo en origen - miembros nacidos en el país de origen del encuestado que residen allí y (4) otros- aquellos miembros cuyo país de residencia no es España ni tampoco el país de origen del encuestado (e.g., familiares que han migrado a otros países que España), y/o cuyo país de origen no es España ni tampoco el país de origen del encuestado (e.g., inmigrantes en España procedentes de otros países que el origen).

También recogimos datos sobre la duración del lazo en años. Si la duración del lazo superó el intervalo de tiempo entre las dos oleadas, entendemos que el miembro fue conocido por la persona encuestada en la primera oleada. Así podemos distinguir entre lazos nuevos (lazos que se han iniciado después de la

EMPIRIA. Revista de Metodología de Ciencias Sociales. N. ${ }^{\circ}$ 26, julio-diciembre, 2013, pp. 63-88. ISSN: 1139-5737, DOI: 10.5944/empiria.26.7153 
primera entrevista) y lazos renovados (lazos con miembros que no se han nombrado en la primera entrevista pero sí existían en aquel momento).

Además, recogimos datos sobre la frecuencia de contacto con cada miembro de la red, en una escala que, recodificada de menor a mayor, va de 1 (una vez al año) a 7 (cada día) y de la proximidad emocional que el encuestado sentía con cada miembro de la red en una escala de 1 (me siento nada próximo) a 5 (muy próximo).

\section{Análisis}

El estudio es de carácter descriptivo. Además de estadísticas descriptivas (como la media, la desviación típica), utilizamos la prueba de $t$, ANOVA y correlaciones (parciales) únicamente para describir las diferencias en el tiempo y entre grupos. Dado el número pequeño de casos y el muestreo no aleatorio, no se usan para probar si las diferencias se pueden generalizar para la población de inmigrantes de estos colectivos en España.

\section{RESULTADOS}

\section{La composición de las redes en la primera entrevista}

Empezamos nuestros análisis con una descripción de las redes de la primera oleada, las cuales constituyen el punto de partida de los cambios descritos a posteriori. En cada oleada, los entrevistados nombraron a 45 personas con los que tenían una relación activa. En la primera oleada, de media 10 de estas 45 personas eran españolas (22\%), 13 (29\%) eran compatriotas residiendo en España, 17 (38\%) eran personas residiendo en el país de origen y $5(11 \%)$ eran personas de otras nacionalidades o residiendo en otros países diferentes del país de origen y de residencia. Por lo tanto, en promedio más de la mitad de la red residía en España en la primera oleada, por lo que se puede considerar que la red es bastante local. Más de la mitad de los miembros de la red que residen en origen y de la categoría «otros» son familiares; entre los compatriotas en España figuran mayoritariamente familiares (un tercio) y personas que han conocido como vecinos (un cuarto). Entre los españoles figuran sobre todo personas que han conocido ${ }^{7}$ como vecinos y compañeros de trabajo del mismo nivel, y en algunos casos compañeros de estudios.

\footnotetext{
${ }^{7}$ Basado en la variable «razón de encuentro». En esta variable, se recoge el contexto de conocer a una persona, no el tipo de relación actual. Las categorías son: esposo/a o pareja, familia por descendencia, familia por matrimonio, alguien con quien trabaja del mismo nivel, alguien para él que trabaja, alguien que trabaja para usted, vecino/a, alguien con el que se encuentra en la iglesia o centro de culto, alguien que conoce a través de alguna asociación o club, alguien con el que se encuentra a causa de una tercera persona, en la escuela, desde la juventud, y otro.
} 
Centrándonos en la fuerza de los lazos, observamos que por término medio un encuestado tiene contacto dos veces al mes con cada miembro de la red $(M=3,1)$ y se siente «bastante próximo» con ellos $(M=3,3$, véase la primera fila de la Tabla 2). Como era de esperar, la fuerza de los lazos difiere entre los cuatro subgrupos en la red. La proximidad emocional que los encuestados sienten es lo más elevada hacia las personas en origen en sus redes, seguido por las compatriotas en destino, los otros y los españoles (véase la Tabla 2). Por lo tanto, los contactos que los encuestados tienen con españoles suelen ser menos próximos que los contactos con los demás miembros de red $\left(M_{\text {españoles }}=2,9 ; D T=0,9 ; M_{\text {no españoles }}\right.$ $=3,5 ; D T=0,7 ; t=7,1, p<, 001)$. Por ejemplo, los encuestados nombraron a 11,2 personas con los que se sentían muy próximas y tan sólo 1,2 de ellas era españolas (11\%), mientras que 3,4 de ellas eran compatriotas en España, 5,2 eran personas en origen y 1,4 otros tipos de personas. A medida que baja la categoría de proximidad, los españoles están cada vez mejor representados $(19 \%, 25 \%$, $32 \%$ y $38 \%$ respectivamente). Por otro lado, la frecuencia de contacto es más elevada entre los lazos locales (correspondiendo a «una vez a la semana», véase la Tabla 2) que entre los lazos lejanos (correspondiendo a «dos veces al año» / «una vez al mes», véase la Tabla $2 ; M_{\text {locales }}=4,2 ; D T=1,0 ; M_{\text {lejanos }}=2,1 ; D T=1,3$; $t=12,6, p<, 001)$.

Describimos a continuación las diferencias interindividuales en el perfil medio esbozado anteriormente. Centrándonos en sexo, observamos que las mujeres tienden a tener casi el doble de personas españolas en su red que los hombres (véase la Tabla 2, «por género»; $t=3,4, p=, 001$ ) y, como consecuencia del tamaño fijo de la red, menos compatriotas en origen y en España. Este resultado se debe a las diferencias entre los países de origen en la proporción de mujeres en la muestra (véase la Tabla 1) y en la composición de las redes (véase abajo), ya que controlando por el país de origen, el efecto de género ya no es estadísticamente significativo. No hay diferencias en género en la proximidad media de los lazos en general, ni en la proximidad media de lazos con los subgrupos en la red. Tampoco hay diferencias en género en la frecuencia de contacto media, aunque observamos que los hombres tienen más contacto que las mujeres con personas de origen español, con compatriotas en España y con personas residentes en o procedentes de otros países (véase la Tabla 2). No obstante, controlando por el país de origen, estos efectos estadísticos de género desaparecen. 


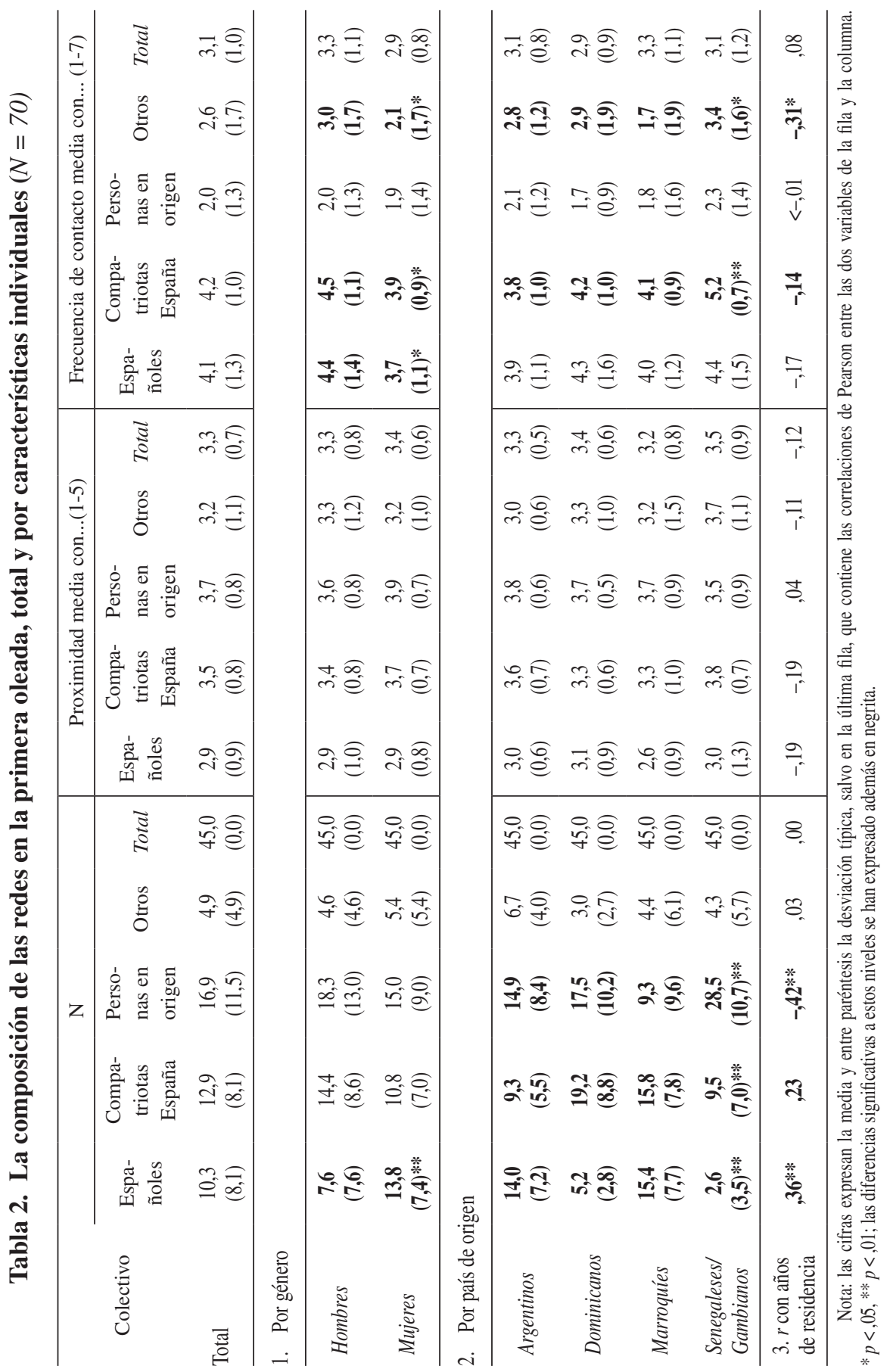

EMPIRIA. Revista de Metodología de Ciencias Sociales. N. ${ }^{\circ}$ 26, julio-diciembre, 2013, pp. 63-88. ISSN: 1139-5737, DOI: 10.5944/empiria.26.7153 
Segundo, se observan grandes diferencias en composición entre los cuatro orígenes en la muestra. Los argentinos y los marroquíes tienen un número de españoles mucho más elevado en la red de la primera oleada (14,0 y 15,4 respectivamente) que los dominicanos y los senegaleses / gambianos en nuestra muestra (5,2 y 2,6 respectivamente, véase la Tabla 2 , «por país de origen»). Por lo tanto, el promedio general de 10 españoles en la red (o un cuarto de la red) no refleja bien la composición reticular de ningún colectivo. El colectivo que se centra más que los otros en los compatriotas en destino son los dominicanos. No obstante, los marroquíes también tienen muy presentes a los compatriotas en destino, por lo que tienen las redes más locales de los cuatro colectivos (con casi un $70 \%$ de relaciones locales). Los senegaleses / gambianos están muy centrados en las personas en origen (63\% de los alteri) y por lo tanto, parecen más marginalizados en España en términos de Berry (2003). No observamos diferencias entre países de origen en la proximidad media de los contactos en general, ni en la proximidad media de los contactos con los subgrupos en la red. Aunque no hay diferencias entre los países de origen en la frecuencia de contacto media, sí observamos diferencias en la frecuencia de contacto con compatriotas en España (los senegaleses los contactan más, los argentinos menos) y con personas en la categoría «otros» (los senegaleses los contactan más, los marroquíes los contactan menos, véase la Tabla 2).

Por último, observamos relaciones entre la composición de las redes y los años de residencia en España. Como se podría esperar, los encuestados con más años de residencia nombran a más españoles y a menos personas en origen que los inmigrantes más recientes (véase la Tabla $2, \ll r$ con años de residencia»). Si categorizamos a la variable años de residencia, observamos que los inmigrantes que residieron durante 2 o menos años en España conocen de media a 5 españoles, los que residieron 3 hasta 5 años en España a 12 españoles y los con 6 o más años de residencia, a 13 españoles. El número de miembros de red en origen para estas categorías es de 26, 16 y 10, respectivamente. El número de compatriotas en España está positivamente pero no significativamente relacionado con el tiempo de residencia y el número de personas en la categoría «otros» no tiene relación con ello. En estudios transversales estas relaciones con los años de residencia se interpretan a menudo como una tendencia temporal, en la que los inmigrantes forman relaciones con españoles y pierden relaciones en origen a medida de la duración de su estancia en España. No obstante, esta relación también puede ser causada por una tercera variable. En nuestro caso, es el colectivo senegalés que influye en cierta medida en estos resultados. El colectivo senegalés tienen un historial de inmigración más reciente que los otros colectivos, lo que está reflejado en nuestra muestra (véase la Tabla 1) y además son los que conocen a menos españoles. Si controlamos nuestro análisis por país de origen, ya no hay una relación estadísticamente significativa entre los años de residencia y el número de españoles en la red, pero sigue haber una relación negativa entre los años de residencia y el número de personas en origen. Este resultado sugiere que los inmigrantes en nuestra muestra pierden relaciones en origen con el tiempo, pero que la medida en la que forman relaciones con españoles depende de

EMPIRIA. Revista de Metodología de Ciencias Sociales. N. ${ }^{\circ}$ 26, julio-diciembre, 2013, pp. 63-88. ISSN: 1139-5737, DOI: 10.5944/empiria.26.7153 
otras características que el tiempo. Constatamos además que los inmigrantes más establecidos no tienen relaciones más fuertes que los inmigrantes más recientes en términos de proximidad emocional y de frecuencia de contacto. Tampoco podemos concluir que los contactos con algún grupo (por ejemplo, los españoles) están, en promedio, más próximos para los que llevan más tiempo de residencia en España, pero la frecuencia con personas en la categoría «otros» está negativamente relacionada con los años de residencia. No obstante, cuando se controla por el país de origen, este efecto desaparece también.

\section{¿En qué medida se renuevan las redes en el corto plazo?}

En total 18,9 de los 45 miembros de red nominados en la primera oleada (es decir, un $42 \%$ ) se nominan de nuevo en la segunda oleada. Los otros 26,1 miembros de red han sido remplazados por otras personas. La variación individual en la estabilidad de la red es considerable: el mínimo número de lazos estables observado en una red es de 10, el máximo es de $34(D T=6,1)$. Como era de esperar, la duración del intervalo de tiempo entre las dos entrevistas está negativamente relacionado con esta estabilidad: a medida que el intervalo entre la primera y la segunda entrevista se hace más largo, menos lazos estables se observan entre la red en la primera oleada y la red en la segunda oleada $(r=-0,49$, $p<, 001)$.

En segundo lugar se analizaron las diferencias en estabilidad por sexo, país de origen y años de residencia (véase la Tabla 3 ). Las redes de las mujeres eran significativamente más estables $(M=21,5$ lazos estables, $D T=6,3)$ que las de los hombres $(M=17,0$ lazos estables, $D T=5,2 ; F=10,5, p<, 01)$, mientras que la duración media del intervalo entre las dos oleadas era igual para los dos grupos. Los argentinos tenían redes más estables que los otros tres colectivos: nombraron de media a 23,5 personas idénticas en las dos oleadas, mientras que este número fue de 16,5 de media para el resto de los colectivos $(F=9,8, p<, 001)$. No obstante, cabe mencionar que la variación interindividual en estabilidad (indicada por la desviación típica) es la más alta entre los argentinos. Sorprendentemente, controlando por las diferencias en el intervalo de tiempo, no se observó una relación entre la duración de residencia en España y la estabilidad de la red ( $r$ parcial $=0,07$, no significativo). En otras palabras, los que han residido en España durante más años tenían redes igual de (in)estables que los que se han trasladado a España más recientemente.

La estabilidad puede parecer baja y por lo tanto es importante destacar que no todos los 26,1 lazos nominados por primera vez en la segunda oleada eran contactos formados después de la primera entrevista: los datos que recogimos sobre la duración de cada relación muestran que los entrevistados ya conocían a 16,9 de ellos antes del momento de la primera entrevista, pero no los nombraron como miembros de la red en aquel momento. Entre otros, pueden ser «lazos durmientes» (contactos que no eran muy activos en aquel momento, pero que luego se revitalizaron) o lazos indirectos (por ejemplo, un amigo de un amigo) que con 
el tiempo se transformaron en relaciones directas. En consecuencia, sólo 9,2 de las 45 relaciones nombradas en la segunda oleada (20\%) eran realmente recién formadas.

La Tabla 3 muestra que las mujeres no difieren mucho de los hombres en cuanto al número de relaciones recién formadas $(F=0,3, n s)$ y que los argentinos destacan entre los colectivos de origen por la cantidad de relaciones recién formadas $(F=7,6, p<, 001)$. No hay una relación significativa con los años de residencia.

Tabla 3. Estabilidad de la red, en total y en relación con género, país de origen y años de residencia

\begin{tabular}{|c|c|c|c|c|}
\hline \multicolumn{2}{|c|}{ Colectivo } & \multirow{2}{*}{$\begin{array}{c}\begin{array}{l}\text { N lazos } \\
\text { estables }\end{array} \\
M(D T)\end{array}$} & \multirow{2}{*}{$\begin{array}{c}\begin{array}{c}\text { N lazos nuevos } \\
\text { con duración } \\
\text { de }>3 \text { años }\end{array} \\
M(D T)\end{array}$} & \multirow{2}{*}{$\begin{array}{c}\begin{array}{c}\text { N lazos nuevos } \\
\text { con duración } \\
\text { de } \leq 3 \text { años }\end{array} \\
M(D T)\end{array}$} \\
\hline & & & & \\
\hline Total & & $18,9(6,1)$ & $16,9(9,6)$ & $9,2(7,0)$ \\
\hline \multirow{2}{*}{ 1. Por género: } & Mujeres & $21,5(6,4)$ & $13,8(10,1)$ & $9,7(6,8)$ \\
\hline & Hombres & $17,0(5,2)$ & $19,3(8,6)$ & $8,7(7,3)$ \\
\hline \multirow{4}{*}{$\begin{array}{l}\text { 2. Por país de } \\
\text { origen: }\end{array}$} & Argentinos & $23,5(6,4)$ & $7,6(8,0)$ & $13,9(7,4)$ \\
\hline & Dominicanos & $17,5(5,0)$ & $22,2(5,6)$ & $5,3(5,6)$ \\
\hline & Marroquíes & $16,5(4,3)$ & $22,2(6,3)$ & $6,3(4,2)$ \\
\hline & $\begin{array}{l}\text { Senegaleses/ } \\
\text { Gambianos }\end{array}$ & $15,7(4,1)$ & $21,0(6,8)$ & $8,3(6,5)$ \\
\hline \multirow{2}{*}{\multicolumn{2}{|c|}{ 3. Años de residencia }} & $\mathrm{r}(\mathrm{p})$ & $\mathrm{r}(\mathrm{p})$ & $\mathrm{r}(\mathrm{p})$ \\
\hline & &,$- 12(\mathrm{~ns})$ & ,22 (ns) &,$- 19(\mathrm{~ns})$ \\
\hline
\end{tabular}

\section{¿En qué medida se altera la composición de las redes?}

Por ahora solamente hemos observado el tamaño del cambio entre las dos oleadas, pero no hemos analizado los contenidos del cambio. Para ello miramos a la composición de la red y a la fuerza de las relaciones. Como ya habíamos indicado en el párrafo anterior, en la segunda oleada un $42 \%$ de las personas nombradas en la primera oleada se vuelven a nominar. Esta estabilidad es algo más elevada entre los compatriotas en España que entre los otros tres tipos de lazos: la estabilidad es de $40 \%$ entre españoles y personas en la categoría «otros», de $41 \%$ entre personas en origen y $46 \%$ entre compatriotas en España. Esto implica que se mantienen de media a 4 españoles, 6 compatriotas en España, 7 personas en origen y 2 otros en la red.

El hecho de que estos 19 miembros de la red se mantengan en la red no quiere decir que las relaciones con ellos no cambien en el tiempo. Con (de media) 
cinco de ellos la relación se hace más fuerte en términos de proximidad emocional y con seis de ellos se hace más débil. Además, hay movilidad geográfica entre los lazos estables. De media hay 0,6 miembros por red que residen en origen y que se trasladan a España en el ínterin y 0,1 que viven en otros países y que se reubican en España. Por otro lado, hay 0,4 miembros que son compatriotas en España regresando al país de origen y 0,3 que son compatriotas en España que se reubican en otros países. También hay de media 0,2 personas residentes en y/o procedentes de otros países que se reubican al país de origen y 0,2 personas en origen que se mueven a otros países. El efecto neto de estos cambios es que el número de miembros de la red en el país de origen disminuye un 0,2 y el número que residen en otros países aumenta un 0,2 .

Como hemos indicado anteriormente, se añaden de media 26,1 nuevas nominaciones. Como acabamos de decir estas nuevas nominaciones no indican relaciones recién formadas. De hecho, solo 9,2 de ellas son relaciones recién formadas, con de media 3,6 españoles (40\%), 3,3 compatriotas en España (36\%), 0,6 persona en origen $(6 \%)$, y 1,7 otros (18\%). Estas cifras muestran que entre las relaciones más recientes, los españoles tienen un peso más grande que en el conjunto de la red. Muchos de estas relaciones nuevas con españoles se forman en el trabajo $(35,9 \%)$ y también a través de otras personas $(17,9 \%)$. Las cifras también indican que el contexto social del origen no es un contexto inactivo, porque incluso (aunque muy poco) se forman nuevas relaciones durante las estancias en origen, muchos a través de otras personas. Las otras 16,9 de las 45 relaciones son relaciones con personas que los encuestados ya conocieron antes de la primera oleada, aunque se supone que no tenían mucha relación con ello en aquel momento. La mayor parte $(57,6 \%)$ de ellos son familiares.

Aunque hay mucha renovación y movimiento al nivel de los alteri, la composición media de las redes no cambia mucho, salvo que unas dos personas en origen han sido remplazadas por compatriotas en destino (véase la Tabla 4, columnas «total»). Este cambio no se puede explicar por reagrupación familiar entre los lazos estables, como hemos indicado anteriormente. Por lo tanto, podemos concluir que aunque las redes se han hecho en promedio un poco más locales (con un aumento medio de dos compatriotas residiendo en España), en general no se observa una tendencia hacia un mayor número de españoles en las redes. Otras medidas, como la frecuencia de contacto y la proximidad emocional con españoles muestran también que de media, las relaciones con españoles o con otros grupos no se han hecho más fuertes con el tiempo. Esto nos indica que aunque hay mucha inestabilidad en las redes, el perfil medio de las redes cambia muy poco en el corto plazo.

A continuación analizamos las tendencias separadas por sexo y por país de origen y analizamos en qué medida hay diferencias entre hombres y mujeres y entre los países de origen en los cambios en su composición.

Primero, las redes de los hombres y mujeres no cambian mucho en el tiempo (véase la Tabla 4, «por género»). Aunque hay diferencias entre hombres y mujeres en la composición de las redes en la primera oleada, el cambio en el tiempo

EMPIRIA. Revista de Metodología de Ciencias Sociales. N. 26, julio-diciembre, 2013, pp. 63-88. ISSN: 1139-5737, DOI: 10.5944/empiria.26.7153 
no se ve afectado por el sexo (es decir, no hay diferencias significativas entre hombres y mujeres en las diferencias en composición entre $t_{1}-t_{2}$ ).

Tabla 4. Comparación de la composición reticular en las dos oleadas, total y por género y correlación entre el cambio en composición y los años de residencia $(N=70)$

\begin{tabular}{|c|c|c|c|c|c|c|c|}
\hline \multirow{3}{*}{$\begin{array}{l}\text { Característica } \\
\text { de la } \\
\text { composición } \\
\text { de la red }\end{array}$} & \multirow{2}{*}{\multicolumn{2}{|c|}{ Total }} & \multicolumn{4}{|c|}{ 1. Por género } & \multirow{3}{*}{$\begin{array}{l}\text { 3. Correlación } \\
\text { parcial entre el } \\
\text { cambio T1-T2 } \\
\text { y los años de } \\
\text { residencia }\end{array}$} \\
\hline & & & \multicolumn{2}{|c|}{ Hombres } & \multicolumn{2}{|c|}{ Mujeres } & \\
\hline & $\mathrm{T} 1$ & $\mathrm{~T} 2$ & $\mathrm{~T} 1$ & $\mathrm{~T} 2$ & $\mathrm{~T} 1$ & $\mathrm{~T} 2$ & \\
\hline \multicolumn{8}{|l|}{$\overline{\mathrm{N}}$} \\
\hline Españoles & 10,3 & 10,1 & 7,6 & 7,7 & 13,8 & 13,3 &,- 05 \\
\hline $\begin{array}{r}\text { Compatriotas } \\
\text { en España }\end{array}$ & 12,9 & $15,3 *$ & 14,4 & 16,6 & 10,8 & 13,5 &,- 16 \\
\hline $\begin{array}{r}\text { Personas en } \\
\text { origen }\end{array}$ & 16,9 & $14,1 *$ & 18,3 & 15,2 & 15,0 & 12,6 & ,18 \\
\hline Otros & 4,9 & 5,5 & 4,6 & 5,5 & 5,4 & 5,6 & ,01 \\
\hline
\end{tabular}

Proximidad media (1-5)

\begin{tabular}{|c|c|c|c|c|c|c|c|}
\hline Españoles & 2,9 & 3,1 & 2,9 & 3,2 & 2,9 & 3,1 & ,09 \\
\hline $\begin{array}{r}\text { Compatriotas } \\
\text { en España }\end{array}$ & 3,5 & 3,5 & 3,4 & 3,4 & 3,7 & 3,5 & ,12 \\
\hline $\begin{array}{r}\text { Personas en } \\
\text { origen }\end{array}$ & 3,7 & 3,8 & 3,6 & 3,8 & 3,9 & 3,8 &,- 01 \\
\hline Otros & 3,2 & 3,3 & 3,3 & 3,4 & 3,2 & 3,1 &, 24 \\
\hline Total & 3,3 & 3,5 & 3,3 & 3,5 & 3,4 & 3,4 & ,06 \\
\hline
\end{tabular}

Frecuencia media (1-7)

\begin{tabular}{|c|c|c|c|c|c|c|c|}
\hline Españoles & 4,1 & 3,8 & 4,4 & 4,0 & 3,7 & 3,5 & , 17 \\
\hline $\begin{array}{r}\text { Compatriotas } \\
\text { en España }\end{array}$ & 4,2 & 4,1 & 4,5 & 4,1 & 3,9 & 4,0 & ,13 \\
\hline $\begin{array}{r}\text { Personas en } \\
\text { origen }\end{array}$ & 2,0 & 2,1 & 2,0 & 2,1 & 1,9 & 2,1 &,- 11 \\
\hline Otros & 2,6 & 2,9 & 3,0 & 3,1 & 2,1 & 2,1 & ,14 \\
\hline Total & 3,1 & 3,3 & 3,3 & 3,3 & 2,9 & 3,2 &,- 07 \\
\hline
\end{tabular}


Tabla 5. Comparación de la composición de las redes en las dos oleadas, por país de origen $(N=70)$

\begin{tabular}{|c|c|c|c|c|c|c|c|c|}
\hline \multirow{3}{*}{$\begin{array}{l}\text { Característica } \\
\text { de la composi- } \\
\text { ción de la red }\end{array}$} & \multicolumn{8}{|c|}{ 2. Por país de origen } \\
\hline & \multicolumn{2}{|c|}{ Argentinos } & \multicolumn{2}{|c|}{ Dominicanos } & \multicolumn{2}{|c|}{ Marroquíes } & \multicolumn{2}{|c|}{$\begin{array}{l}\text { Senegaleses/ } \\
\text { Gambianos }\end{array}$} \\
\hline & $\mathrm{T} 1$ & $\mathrm{~T} 2$ & $\mathrm{~T} 1$ & $\mathrm{~T} 2$ & $\mathrm{~T} 1$ & $\mathrm{~T} 2$ & $\mathrm{~T} 1$ & $\mathrm{~T} 2$ \\
\hline \multicolumn{9}{|l|}{$\mathrm{N}$} \\
\hline Españoles & 14,0 & 15,6 & 5,2 & 4,8 & 15,4 & 11,8 & 2,6 & 3,8 \\
\hline $\begin{array}{l}\text { Compatriotas } \\
\text { en España }\end{array}$ & 9,3 & 8,0 & 19,2 & 24,2 & 15,8 & 18,8 & 9,5 & $14,9 *$ \\
\hline $\begin{array}{l}\text { Personas en } \\
\text { origen }\end{array}$ & 14,9 & 14,7 & 17,5 & $11,2 *$ & 9,3 & 8,8 & 28,5 & $21,9 * *$ \\
\hline Otros & 6,7 & 6,7 & 3,0 & 4,8 & 4,4 & 5,6 & 4,3 & 4,3 \\
\hline \multicolumn{9}{|l|}{$\begin{array}{l}\text { Proximidad } \\
\text { media (1-5) }\end{array}$} \\
\hline Españoles & 3,0 & 3,2 & 3,1 & 3,1 & 2,6 & 2,9 & 3,0 & 3,6 \\
\hline $\begin{array}{l}\text { Compatriotas } \\
\text { en España }\end{array}$ & 3,6 & $3,8^{*}$ & 3,3 & 3,6 & 3,3 & 3,1 & 3,8 & $3,4^{*}$ \\
\hline $\begin{array}{l}\text { Personas en } \\
\text { origen }\end{array}$ & 3,8 & 4,0 & 3,7 & $4,3 * *$ & 3,7 & $3,1^{*}$ & 3,5 & 3,9 \\
\hline Otros & 3,0 & $3,4^{*}$ & 3,3 & 3,2 & 3,2 & 3,0 & 3,7 & 3,5 \\
\hline Total & 3,3 & $3,6^{*}$ & 3,4 & 3,6 & 3,2 & 3,1 & 3,5 & 3,6 \\
\hline \multicolumn{9}{|l|}{$\begin{array}{l}\text { Frecuencia } \\
\text { media (1-7) }\end{array}$} \\
\hline Españoles & 3,9 & 3,5 & 4,4 & 3,3 & 4,0 & 3,8 & 4,4 & $5,4^{*}$ \\
\hline $\begin{array}{l}\text { Compatriotas } \\
\text { en España }\end{array}$ & 3,8 & 3,9 & 4,2 & 4,0 & 4,1 & 4,0 & 5,2 & 4,6 \\
\hline $\begin{array}{l}\text { Personas en } \\
\text { origen }\end{array}$ & 2,1 & 1,7 & 1,7 & 2,4 & 1,8 & 2,0 & 2,3 & 2,7 \\
\hline Otros & 2,8 & 3,0 & 2,9 & 3,7 & 1,7 & 1,8 & 3,4 & 3,5 \\
\hline Total & 3,1 & 3,0 & 2,9 & $3,5^{*}$ & 3,3 & 3,2 & 3,1 & 3,7 \\
\hline
\end{tabular}

$* p<, 05, * * \quad p<, 01 ;$ las diferencias significativas a estos niveles se han expresado además en negrita.

Segundo, si miramos a las tendencias separadas por país de origen (véase la Tabla 5), se observa que los argentinos, un grupo que ya tenía relativamente mu-

EMPIRIA. Revista de Metodología de Ciencias Sociales. N. o 26, julio-diciembre, 2013, pp. 63-88. ISSN: 1139-5737, DOI: 10.5944/empiria.26.7153 
chas relaciones con españoles en la primera entrevista, manifiesta pocos cambios en la composición de sus redes. El aumento en el número de compatriotas en España y el descenso en el número de personas en origen que observamos en general no se observan en este colectivo. Solamente se observa que los argentinos en nuestra muestra se sienten más próximos con los miembros de sus redes en general y específicamente con la categoría denominada «otros». No hay cambios significativos en el tiempo en el número de personas en cada subgrupo ni en la frecuencia de contacto. Surge la pregunta si este resultado significa que hay poco cambio en las redes individuales en el tiempo, quizás por el intervalo pequeño entre las dos oleadas, o más bien que el cambio en algunas redes anula el cambio en la dirección opuesta en otras. Efectivamente, para los argentinos observamos básicamente dos perfiles individuales opuestos: los que han aumentado su número de contactos con españoles (y por lo tanto, han perdido relaciones en otros grupos) y los que han reactivado los contactos en origen, a coste de los contactos en destino. El segundo perfil parece ser una involución, pero las razones que los inmigrantes proporcionaron acerca de la reactivación de contactos en origen en la parte cualitativa de la entrevista indican a menudo una inestabilidad temporal en la red, debido a viajes recientes al país de origen y a la remigración de compatriotas que antes vivieron en España. Es posible que esta inestabilidad se normalice en el corto plazo.

El perfil medio de los marroquíes tampoco cambia mucho en el tiempo, aunque se nota en promedio un aumento en el número de compatriotas en España (de 3 contactos) y una disminución en el número de españoles en la red (de 3,6 contactos). Los marroquíes son el único colectivo que por término medio pierde relaciones con españoles. No hay grandes cambios en la fuerza de las relaciones, salvo que los marroquíes se sienten menos próximos con las personas en origen. Una vez más nos preguntamos si el promedio refleja bien el perfil de cambio en las redes individuales. Así, hemos observado varios perfiles de cambio entre los marroquíes: los que han ido concentrándose a los contactos con compatriotas en España y han perdido algunas relaciones con españoles; los que han reactivado (temporalmente o no) las relaciones en origen, también a coste de los españoles y los que han centrado más en sus otros contactos (residentes de otros países que origen y destino o inmigrantes procedentes de otros países que origen y destino).

Los cambios más destacados en el tiempo son el aumento en el número de compatriotas en España y el descenso simultáneo del número de personas en origen por parte de los senegaleses y gambianos y de los dominicanos, lo que influye en la tendencia general. En los dos casos había relativamente pocos españoles en la red en la primera y en la segunda oleada. Los dominicanos tenían muchos compatriotas en sus redes en la primera oleada, tanto en origen como en España. Como resultado del cambio entre las oleadas, los dominicanos ahora están más orientados a los compatriotas en España. La pérdida de relaciones en origen va asociada con un aumento en la percepción de proximidad con los miembros de la red en origen y el aumento del contacto de frecuencia con ellos, lo que sugiere que se han perdido las relaciones más débiles en origen (o que se mantienen las más fuertes).

EMPIRIA. Revista de Metodología de Ciencias Sociales. N. ${ }^{\circ}$ 26, julio-diciembre, 2013, pp. 63-88. ISSN: 1139-5737, DOI: 10.5944/empiria.26.7153 
Aunque los senegaleses y senegambianos nombran bastante menos relaciones en origen que en la primera oleada, siguen caracterizándose por el número de personas en origen ya que tenían muchas relaciones en origen en la primera oleada. El aumento de compatriotas en España va asociado con un descenso en la proximidad que los encuestados senegaleses y gambianos sienten hacia los compatriotas en España, lo que sugiere que han añadido lazos relativamente débiles con compatriotas en España. La disminución en el número de personas en origen va asociado con un aumento en proximidad que sienten hacia ellas, lo que sugiere igualmente que han perdido relaciones débiles en origen a cambio de relaciones débiles en destino.

La medida en que la composición cambia en el tiempo difiere entre los diferentes orígenes. Más específicamente, hay diferencias en el cambio en el número de españoles en la red $(F=3,1, p<, 05)$, el número de compatriotas en España $(F=2,9, p<, 05)$ y de personas en origen $(F=2,9, p<, 05)$, en la proximidad que los encuestados sienten hacia los personas en origen $(F=6,0$, $p=, 001)$ y en destino $(F=3,9, p<, 05)$ y en la frecuencia de contacto con los españoles $(F=4,3, p<, 01)$.

Sorprendentemente, los años de residencia no correlacionan significativamente con ninguno de los cambios en la composición en el tiempo (ni linealmente ni cuadráticamente), tanto cuando se controla por el intervalo de tiempo como cuando no (véase la Tabla 4 , «Correlación parcial cambio $t_{1}-t_{2}$ con años de residencia», para las correlaciones lineales parciales, corregidas por el intervalo de tiempo). Esto implica que los años de residencia no afectan de forma directa a los cambios en las redes recogidas en nuestro estudio.

\section{CONCLUSIÓN}

En el presente artículo hemos descrito cómo se reconstruyen las redes personales a corto plazo en el trayecto postmigratorio. Como hemos indicado en la introducción, las redes personales pueden ser una fuente de apoyo social esencial para el inmigrante, pero es una fuente dinámica y la medida en la que puede proporcionar apoyo depende entre otros factores de la composición de la red en cada momento. Teníamos dos preguntas primero, en promedio y sobre el período mencionado anteriormente, ¿en qué medida se renuevan las redes en el corto plazo? Segundo, ¿en promedio, cómo cambia la composición de las redes en términos de lugar de origen y de residencia de los miembros de la red y de la fuerza de los lazos en este período?

Para responder a estas preguntas, hemos realizado un estudio sobre el cambio en las redes personales activas de 77 inmigrantes en la provincia de Barcelona sobre un intervalo de tiempo de 2 años de media. Los inmigrantes proceden de cinco países: argentinos, dominicanos, marroquíes y senegaleses y gambianos. El diseño longitudinal y la medición de redes amplias (de 45 personas cada una) nos han permitido seguir el cambio con mucha detalle. 
Primero, observamos que el volumen de cambio en las redes es bastante alto. Sólo 19 de los 45 miembros de la red mencionados en la primera entrevista han sido nominados por segunda vez en la segunda entrevista (42\%). No obstante, de las 26 relaciones que fueron nominadas por primera vez en la segunda oleada, 17 ya eran conocidos por los encuestados en la primera oleada, aunque en aquel momento quizás no formaban parte de su red activa. En muchos casos se trata de relaciones «durmientes», que se han revitalizado en la segunda entrevista (como por ejemplo lazos con familiares lejanos) y por otro lado de relaciones indirectas (por ejemplo, un amigo de un amigo) que se han hecho directas con el tiempo. Por lo tanto, entre las dos oleadas, solamente 9 de las 45 relaciones de media (un 20\%) aparecieron en los últimos dos años. Mientras que el porcentaje de españoles en la red es de $22 \%$ de media, el porcentaje de españoles entre los miembros nuevos de la red es casi el doble (40\%). Aún si tomamos en cuenta que los lazos nuevos suelen ser débiles y la estabilidad de estas relaciones es más baja que la de los lazos fuertes, este alto porcentaje de españoles en los lazos nuevos es destacable, lo que podría sugerir que la incorporación de lazos estables con españoles precisa de tiempo. Sorprendentemente, el volumen del cambio total no depende de los años de residencia en España, lo que significa sencillamente que las redes de los inmigrantes recientes cambiaron en igual medida que las de los inmigrantes más establecidos.

Segundo, hemos analizado las tendencias medias del cambio de las redes en el tiempo, primero para el total de los encuestados y en segundo lugar separado por sexo, país de origen y años de residencia. A pesar de la considerable inestabilidad en las redes a nivel de los alteri, observamos que la composición media de las redes cambia muy poco con el tiempo, salvo un ligero aumento en el número de compatriotas residiendo en España y una ligera disminución del número de personas residiendo en el país de origen del encuestado. Esto indica que las redes se han hecho ligeramente más locales, especialmente entre los dominicanos y senegambianos, que han incorporado a más compatriotas en España. Como consecuencia, los dominicanos y los senegaleses están concentrando las funciones de la red mayoritariamente en los compatriotas, aunque en el caso de los senegaleses, con su trayectoria más reciente de inmigración (en nuestra muestra), en ambos oleadas la mayoría de los alteri siguen residiendo en origen. Los argentinos muestran una de las dos tendencias: bien la incorporación de españoles, bien la reactivación de lazos en origen. Esta última tendencia parece indicar una inestabilidad temporal. Los marroquíes también tienen varios perfiles, pero ninguno de ellos implica la incorporación de españoles. Al contrario, los marroquíes pierden relaciones con españoles con el tiempo. Por término medio, no hay un aumento en el número de españoles en la red, ni observamos que las relaciones con españoles se hagan más fuertes con el tiempo. Las tendencias son parecidas para hombres y mujeres y no dependen de los años de residencia en España.

Por supuesto, este estudio presenta importantes limitaciones. En primer lugar la muestra no es aleatoria y el reducido número de casos estudiado no nos permite generalizar las conclusiones alcanzadas, sino solamente apuntar hipótesis de futuro que precisarán investigaciones diseñadas ad hoc para poder llegar a resultados concluyentes. Por otra parte, el tiempo transcurrido entre las dos olea-

EMPIRIA. Revista de Metodología de Ciencias Sociales. N. ${ }^{\circ}$ 26, julio-diciembre, 2013, pp. 63-88. ISSN: 1139-5737, DOI: 10.5944/empiria.26.7153 
das, aún siendo significativo, es demasiado corto para poder apreciar cambios de más largo alcance. Actualmente estamos terminando la recogida de una tercera oleada de datos, 6-8 años después de la primera oleada, y esperamos que este intervalo nos permitirá estudiar a la reconstrucción de la red a medio plazo. Por último, afirmamos que hace falta complementar estos resultados descriptivos con un estudio más profundo de los mecanismos micro de la dinámica en las redes, es decir, al nivel de las relaciones, en el que también se puede tomar en cuenta la estructura de la red. En este primer estudio, no nos hemos enfocado a la estructura de la red porque no hay bases para suponer que características agregadas de la estructura (como la densidad) cambian de forma lineal en el tiempo para los inmigrantes. No obstante será interesante entender la intersección de características relacionales, individuales y contextuales en la producción de los cambios aquí descritos.

\section{BIBLIOGRAFÍA}

AROIAN, K. J. (1992): «Sources of social support and conflict for Polish immigrants» Qualitative Health Research, 2, 178-207.

ÁVILA MOLERO, J. (2008): «Redes personales de africanos y latinoamericanos en Cataluña, España. Análisis reticular de integración y cambio», REDES-Revista hispana para el análisis de redes sociales, Vol. 15,\#5.

BERRY, JOHN (2003). «Conceptual Approaches to Acculturation». En: Chun, Kevin M. et. al., Acculturation. Advances in Theory, Measurement and Applied Research. Washington DC: American Psychological Association.

CARNET, P. (2011). «Estrategias de activación y de construcción de redes sociales en la migración. El ejemplo de los migrantes africanos clandestinizados en la frontera sur española». REDES-Revista hispana para el análisis de redes sociales, Vol. 20,\#10.

DE MIGUEL LUKEN, V. \& TRANMER, M. (2010). «Personal support networks of immigrants to Spain: A multilevel analysis». Social Networks, 32, 253-262.

DOMÍNGUEZ, S. \& MAYA JARIEGO, I. (2008): «Acculturation of Host Individuals: Immigrants and Personal Networks», American Journal of Community Psychology, 42, 309-327.

DUNBAR R. I. M., \& SPOOR M. (1995): «Social networks, support cliques and kinship», Humane Nature, 6, 273-290.

EVE, M. (2010): «Integrating via networks: Foreigners and others», Ethnic and Racial Studies, 33, 1231-1248.

FELD, S. (2004): «The focused organization of social ties», American Journal of Sociology, 86, 1015-1035.

GILL, N., \& BIALSKI, P. (2011): «New Friends in New Places: Network Formation during the Migration Process Among Poles in the UK», Geoforum, 42(2), 241-249.

GRANOVETTER, M. (1973): «The strength of weak ties», American Journal of Sociology, 78, 1360-1380.

HAGAN, J. (1998): «Social networks, gender, and immigrant corporation: Resources and constraints», American Sociological Review, 63, 55-67.

HONDAGNEU-SOTELO, P., \& CRANFORD, C. (1999), «Gender and Migration,» Pp. 105-126 en Saltzman Chaffetz, J. (Ed), Handbook of the Sociology of Gender. New York: Kluwer Academic/Plenum Publishers. 
KÜRTOSI, Z. (2008): «Differences in female and male social networks in a work setting». Tesis doctoral, Universidad de Budapest.

LIU (2011): «Migrant networks and international migration: Testing weak ties», Paper presented at the PAA 2011.

LUBBERS M.J., MOLINA, J. L., LERNER, J., BRANDES, U., MCCARTY, C. \& ÁVILA, J. (2010): «Longitudinal analysis of personal networks. The case of Argentinean migrants in Spain», Social Networks, 32, 91-104.

LUBBERS, M. J., MOLINA, J. L., \& MCCARTY, C. (2007): «Personal Networks and Ethnic Identifications: The Case of Migrants in Spain», International Sociology, Vol. 22 (6), 720-740.

LYNAM, M. J. (1985): «Support networks developed by immigrant women», Social Science and Medicine, 21 (3), 327-333.

MAHLER, S. (1995): «American Dreaming: Immigrant Life on the Margins», Princeton NJ, Princeton University Press.

MARSDEN, P. (1987): «Core discussion networks of Americans», American Sociological Review, 52, 122-131.

MARSDEN, P. V., \& CAMPBELL, K. E. (1984): «Measuring tie strength», Social Forces, 63, 482-501.

MARSDEN, P. V. \& CAMPBELL, K. E. (2012): «Reflections on conceptualizing and measuring tie strength», Social Forces, 91, 17-23.

MARTINOVIC, B. (2009): «Changes in immigrants' social integration during the stay in the host country: The case of non-Western immigrants in the Netherlands», Social Science Research, 38, 870-882.

MARTINOVIC, B. (2013): «The Inter-Ethnic Contacts of Immigrants and Natives in the Netherlands: A Two-Sided Perspective», Journal of Ethnic and Migration Studies, 39, 69-85.

MAYA JARIEGO, I., MARTÍNEZ, M. F., \& GARCÍA, M. (1999): «Cadenas migratorias y redes de apoyo social de las mujeres peruanas en Sevilla», Demófilo: Revista de Cultura Tradicional de Andalucía, 29, 87-105.

MCCARTY, C. (2002): «Structure in Personal Networks», Journal of Social Structure. <http://www.library.cmu.edu:7850/JoSS/McCarty/McCarty.htm>

MCCARTY, C., MOLINA, J. L., AGUILAR, C. \& L. ROTA (2007): «A Comparison of Social Network Mapping and Personal Network Visualization», Field Methods, Vol. 19 (2), 145-162.

MENJÍVAR, C. (1997): «Immigrant kinship networks and the impact of the receiving context: Salvadorans in San Francisco in the early 1990s», Social Problems, 44.

MOLINA, J.L., BOLÍBAR, M., \& CRUZ, I. (2011): «La dispersión geográfica de las redes personales. Cálculo y significado», REDES-Revista Hispana para el Análisis de Redes Sociales, Vol 20 \#5, 113-131.

MOLINA, J. L., LERNER, J., \& GÓMEZ MESTRES, S. (2008): «Patrones de cambio de las redes personales de inmigrantes en Cataluña», REDES-Revista hispana para el análisis de redes sociales, Volumen 15 \#4, 36-60.

MOLINA, J. L., C. MCCARTY, C. AGUILAR \& ROTA, L. (2007): «La estructura social de la memoria», en LOZARES, C. (Ed.) Interacción, Redes Sociales y Ciencias Cognitivas, Barcelona: La Razón Áurea.

PALLONI, A., \& MASSEY, D. S. (2001): «Social capital and international migration: A test using information on family networks», American Journal of Sociology, 106, 1262-1298. 
PFEFFER, M. J., \& PARRA, P. A. (2009): «Strong Ties, Weak Ties, and Human Capital: Latino Immigrant Employment Outside the Enclave», Rural Sociology, 74, 241-269.

POROS, M. (2011): «Modern migrations: Gujarati Indian networks in New York and London». Stanford University Press, 2011.

PORTES, A. (1995): «Economic sociology and the sociology of immigration: A conceptual overview». In Portes, A. (Ed.) The Economic Sociology of Immigration. New York, Russell-Sage Foundation, pp 1-41.

PORTES, A., \& SENSENBRENNER, J. (1993): «Embeddedness and Integration», American Journal of Sociology, 98, 1320-1350.

ROSE, D., CARRASCO, P., \& CHARBONEAU, J. (1998), «The role of "weak ties" in the settlement experiences of immigrant women with young children: The case of Central Americans in Montréal». Working paper of the Centre of Excellence for Research on Immigration and Settlement (CERIS), Toronto.

RYAN, L. (2011): «Migrants' social networks and weak ties: Accessing resources and constructing relationships post-migration», The Sociological Review, 59, 707-724.

SANDERS, J., NEE, V., \& STERNAU, S. (2002): «Asian immigrants' reliance on social ties in a multi-ethnic labor market», Social Forces, 81, 281-314.

STANTON-SALAZAR, R. D., \& DORNBUSCH, S. M. (1995): «Social capital and the reproduction of inequality: Information networks among Mexican-origin high school students», Sociology of Education, 68, 116-135.

SZELL, M. \& TURNER, S. (2013): «How women organize social networks different from men», Scientific Reports, 3, 1214 (doi: 10.1038/srep01214)

VACCA, R. (2013): «Bridging across nations. The social capital of diversity, brokerage and closure in transnational migrant networks: A study on assimilation patterns in Milan and Barcelona». Unpublished doctoral thesis, Università degli Studi di Milano-Bicocca.

VALENTA, M. (2009): «Finding friends after resettlement: Social networks and everyday life of immigrants in Norway». VDM Verlag.

VERVOORT, M. (2012): «Ethnic concentration in the neighbourhood and ethnic minorities' social integration: Weak and strong social ties examined», Urban Studies, 49, 897-915.

WELLMAN, B. \& WORTLEY, S. (1990): «Different strokes from different folks: Community ties and social support», American Journal of Sociology, 96, 558-588.

WIERZBICKI, S. (2004): «Beyond the immigrant enclave. Network change and assimilation», New York: LFB Scholarly Publishing.

WILSON, T. D. (1998): «Weak ties, strong ties: Network principles in Mexican migration», Human Organization, 57, 394-403.

ZHOU, W.-X., SORNETTE, D., HILL, R. A., \& DUNBAR, R. I. M. (2005): «Discrete hierarchical organization of social group sizes», Proc Biol Sci., 272(1561), 439-444. 